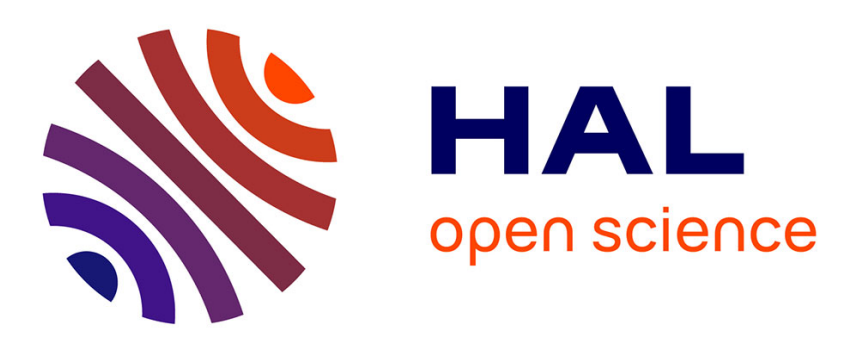

\title{
Turbulence modeling of the Von Karman flow: viscous and inertial stirrings
}

Sébastien Poncet, Roland Schiestel, Romain Monchaux

\section{To cite this version:}

Sébastien Poncet, Roland Schiestel, Romain Monchaux. Turbulence modeling of the Von Karman flow: viscous and inertial stirrings. International Journal of Heat and Fluid Flow, 2008, 29 (1), pp.62-74. 10.1016/j.ijheatfluidflow.2007.07.005 . hal-00202922

\section{HAL Id: hal-00202922 \\ https://hal.science/hal-00202922}

Submitted on 8 Jan 2008

HAL is a multi-disciplinary open access archive for the deposit and dissemination of scientific research documents, whether they are published or not. The documents may come from teaching and research institutions in France or abroad, or from public or private research centers.
L'archive ouverte pluridisciplinaire $\mathbf{H A L}$, est destinée au dépôt et à la diffusion de documents scientifiques de niveau recherche, publiés ou non, émanant des établissements d'enseignement et de recherche français ou étrangers, des laboratoires publics ou privés. 


\title{
Turbulence modeling of the Von Kármán flow: viscous and inertial stirrings
}

\author{
Sébastien Poncet* \\ MSNM-GP, UMR 6181 CNRS-Aix-Marseille Universités \\ IMT La Jetée, Technopôle Château-Gombert, 38 rue F. Joliot-Curie, \\ 13451 Marseille cédex 20 - FRANCE - Fax. 33.4.91.11.85.02 \\ Roland Schiestel ${ }^{\dagger}$ \\ IRPHE, UMR 6594 CNRS-Universités d'Aix-Marseille I \& II \\ Technopôle Château-Gombert, 49 rue F. Joliot-Curie, B.P. 146, \\ 13384 Marseille cédex 13 - FRANCE - Fax. 33.4.96.13.97.09 \\ Romain Monchaux ${ }^{\ddagger}$ \\ Service de Physique de l'État Condensé, \\ DSM/DRECAM/SPEC, CNRS/SPM/URA 2464, \\ CEA Saclay F-91191 Gif sur Yvette Cedex - FRANCE
}

(Dated: June 14, 2007)

The present work considers the turbulent Von Kármán flow generated by two counter-rotating smooth flat (viscous stirring) or bladed (inertial stirring) disks. Numerical predictions based on one-point statistical modeling using a low Reynolds number second-order full stress transport closure (RSM model) are compared to velocity measurements performed at CEA (Commissariat à l'Énergie Atomique, France). The main and significant novelty of this paper is the use of a drag force in the momentum equations to reproduce the effects of inertial stirring instead of modelling the blades themselves. The influences of the rotational Reynolds number, the aspect ratio of the cavity, the rotating disk speed ratio and of the presence or not of impellers are investigated to get a precise knowledge of both the dynamics and the turbulence properties in the Von Kármán configuration. In particular, we highlighted the transition between the merged and separated boundary layer regimes and the one between the Batchelor [1] and the Stewartson [2] flow structures in the smooth disk case. We determined also the transition between the one cell and the 
two cell regimes for both viscous and inertial stirrings.

Keywords: Von Kármán flow, turbulence modeling, LDV, viscous stirring, inertial stirring.

*Electronic address: poncet@13m.univ-mrs.fr, Tel.33.4.91.11.85.23

$\dagger$ Electronic address: schiestel@irphe.univ-mrs.fr, Tel.33.4.96.13.97.65

${ }_{\ddagger}^{\ddagger}$ Electronic address: romain.monchaux@cea.fr, $\mathrm{Tel}$.33.1.69.08.72.14 


\section{INTRODUCTION}

The flow between two finite counter-rotating disks enclosed by a cylinder, known as the Von Kármán [3] geometry, is of practical importance in many industrial devices. Counterrotating turbines may indeed be used to drive the counter-rotating fans in gas-turbine aeroengines. Moreover, this configuration is often used for studying fundamental aspects of developed turbulence and especially of magneto-hydrodynamic turbulence.

From an academic point of view, the laminar flow between two infinite disks has indeed justified many works since the beginning of the controversy between Batchelor [1] and Stewartson [2] on the flow structure. Batchelor [1] solved the system of differential equations relative to the steady rotationally-symmetric viscous flow between two infinite disks. In the exactly counter-rotating regime, the distribution of tangential velocity is symmetrical about the mid-plane and exhibits five distinct zones: two boundary layers developed on each disk, a transition shear layer at mid-plane, where the axial and tangential velocities change sign and two rotating cores on either side of the transition layer. The central cores rotate with a tangential velocity proportional to the disk velocities. The proportionality coefficient is always inferior to 1 . This solution can be regarded as the connection of two Batchelor flows in the rotor-stator configuration. As stated by Batchelor [1] himself, "this singular solution may not be realizable experimentally, of course", which supposes that another solution may exist. In 1953, Stewartson [2] found that the flow is divided into only three zones for large values of the Reynolds number $R e_{H}=\Omega H^{2} / \nu>100$ based on the interdisk space $H(\Omega$ is the rotation rate of the disks and $\nu$ the kinematic viscosity of the fluid): one boundary layer on each disk separated by a zone of zero tangential velocity and uniform radial inflow. Lance and Rogers [4] found numerically in the exactly counter-rotating regime a Stewartson solution for $R e_{H}=1023$. The existence at large Reynolds numbers of the Stewartson solution has been confirmed by the analysis of McLeod and Parter [5] in an infinite counter-rotating disk system. The Stewartson solution has also been obtained numerically by Pesch and Rentrop [6] at $R e_{H}=2000$. Kreiss and Parter [7] have proved the existence and non-uniqueness of solutions at sufficiently large Reynolds numbers for the two-disk configuration. Thus, both Batchelor and Stewartson solutions are possible depending on the initial and boundary conditions but the Batchelor prediction has not been mentioned in the literature for the exact counter-rotating disk case. Pearson [8] obtained numerically a basic inviscid solution 
of the Von Kármán flow, which differs from both the Batchelor and Stewartson solutions: at high Reynolds number $\left(R e_{H}=10^{3}\right)$, the solution is unsymmetrical and the main body of the fluid rotates faster than that of either disk. In the counter-rotating regime, Dijkstra and Van Heijst [9] showed numerically that the transition from the one cell to the two cell structure occurs for a given Reynolds number and corresponds to the appearance of a detached shear layer on the slower disk. Recently, Yang and Liao [10] solved the Von Kármán swirling viscous flow using the homotopy analysis method. The reader is referred to the work of Holodniok et al. [11] and to the review of Zandbergen and Dijkstra [12] for a more extensive survey until 1987.

In the turbulent case, the Von Kármán flow is a model flow to study the turbulence characteristics on small scales. The main flow is axisymmetric and so offers an interesting intermediate situation between two-dimensional and three-dimensional flows. Fauve et al. [13] reported measurements of pressure fluctuations in the turbulent Von Kármán flow. They showed that the pressure probability function is strongly non-Gaussian and displays an exponential tail toward low pressure. Maurer et al. [14] used low-temperature helium gas to obtain high Reynolds numbers and well-defined scaling properties. They established the turbulence characteristics such as structure functions or the probability density function of the velocity differences and confirmed that turbulence on small scales has universal properties independent of the forcing. Mordant et al. [15] investigated the dynamical behavior of the Von Kármán flow at moderate to high Reynolds numbers using spatially averaged measurements. Data of the power input and of pressure fluctuations at the wall are sufficient to calculate the main turbulence characteristics such as the velocity fluctuations or the typical length scales. Cadot et al. [16] measured the mean rates of energy injection and energy dissipation in steady regimes of turbulence in the flow between counter-rotating stirrers. The smooth stirrers are found to be less efficient in setting the fluid into motion than in the case of bladed disks. Pinton et al. [17] measured the power consumption of the turbulent Von Kármán flow at constant Reynolds number and showed that power fluctuations occur and involve coherent fluid motions in the whole cell. Marié and Daviaud [18] performed full velocity measurements linking velocity fluctuations with the turbulent drag in this geometry. They showed especially that the turbulent drag is dominantly generated by coherent structures at the largest scales of the flow. Cadot and Le Maître [19] considered the turbulent between two co- and counter-rotating stirrers. They measured the instanta- 
neous torques driving the flow and compared them to similarity laws having no dependence on the Reynolds number with a good agreement.

Ravelet et al. [20,21] reported experimental evidence of a global bifurcation on a highly turbulent flow between two counter-rotating impellers. The transition between the symmetric and the unsymmetric solutions is subcritical and the system keeps a memory of its history. Monchaux et al. [22] investigated the properties of the mean and most probable velocity fields in the same configuration. They showed that these two fields are described by two families of functions [23] depending on both the viscosity and the forcing. For large values of the Reynolds number, in some regions, the flow behaves like a Beltrami flow in which vorticity is locally aligned with velocity. Boroński [24] simulated the laminar Von Kármán flow between two counter-rotating disks equipped or not by straight blades. For a rotational Reynolds number $R e=\Omega R^{2} / \nu$, based on the disk radius $R$, equal to 500 , the poloidal-to-toroidal ratio is increased from $13 \%$ in the smooth disk case to $51 \%$ in the bladed disk case.

A renewal of interest for the Von Kármán flow is born from the dynamo experiments. The flow between counter-rotating impellers is considered as a possible candidate for the observation of a homogeneous fluid dynamo less constrained than the Riga and Karlsruhe devices. The flow needs to be highly turbulent in order for nonlinearities to develop in the magnetic induction. Numerous experimental [25, 26] or numerical [27, 28] studies have then been dedicated to magneto-hydrodynamics turbulence in the Von Kármán geometry. In the latter work, the flow has been optimized using a water model experiment, varying the driving impeller configuration, well described in [21].

To our knowledge, only very few numerical works have been devoted to the characterization of the mean and turbulent flow properties in the Von Kármán geometry. Kilic et al. [29] performed a combined numerical and experimental study of the transitional flow between smooth counter-rotating disks with a central hub for $-1 \leq \Gamma \leq 0, R e=10^{5}$ and $G=H / R=0.12$, where $\Gamma$ is the ratio between the rotating speeds of the two disks and $G$ is the aspect ratio of the cavity. They compared mean radial and tangential velocity measurements using a single-component laser Doppler anemometer with computed results either the low-Reynolds number $k-\epsilon$ turbulence model of Launder and Sharma [30] or a laminar elliptic code. For $\Gamma=-1$, the weakly turbulent flow is of Stewartson type, whereas the laminar computations and measurements produce a Batchelor type of flow. The tran- 
sitions from laminar to turbulent regime and from Batchelor to Stewartson flow structure occur for $\Gamma=-0.4$. A good agreement is obtained in the rotor-stator configuration $(\Gamma=0)$ and in the exactly counter-rotating regime $(\Gamma=-1)$ but at intermediate values of $\Gamma$, the agreement is less satisfactory. The same authors [31] performed the same comparisons when a radial outflow of air is superimposed.

In this paper, we present comparisons between numerical predictions using a Reynolds Stress Model, denoted RSM, and velocity measurements performed at CEA for the turbulent flow between two counter-rotating disks. The main objective is to acquire a precise knowledge of both the flow structure and the turbulence properties of the high turbulent Von Kármán flow between smooth disks for a large range of the flow control parameters. A second objective is to propose an easy and efficient way to model impellers and to quantify their effect on the Von Kármán flow at high Reynolds number.

\section{EXPERIMENTAL SET-UP}

Velocity measurements using a laser Doppler velocimeter have been performed at CEA in the Von Kármán geometry during the PhD thesis of Ravelet [21] and then by Romain Monchaux [22] in two cases: viscous and inertial stirrings.

\section{A. Geometrical configuration}

We consider the Von Kármán flow generated by two counter-rotating disks fitted or not by straight blades in a cylindrical vessel, as illustrated in figures 1a,b. The geometrical parameters are fixed by the values studied experimentally by Ravelet [21]. The cylinder and disk radii are respectively $R_{c}=100 \mathrm{~mm}$ and $R=92.5 \mathrm{~mm}$. The radius ratio $R / R_{c}$ is then fixed to 0.925 . The distance between the inner faces of the disks $H$ can vary between 1 and $180 \mathrm{~mm}$. Disks 1 and 2 rotate respectively clockwise and counterclockwise with two rotation rates denoted $\Omega_{1}$ and $\Omega_{2}$. The motor rotation rates can be varied independently in the range $0-900 \mathrm{rpm}$, with $\left|\Omega_{1}\right| \geq\left|\Omega_{2}\right|$. We use bladed disks ( $n$ blades of height $h$ equal to 10 or $20 \mathrm{~mm}$ ) to ensure inertial stirring or flat disks for viscous stirring. The impellers are driven by two independent brushless $1.8 \mathrm{~kW}$ motors, with speed servo loop control. 


\section{B. Measurement technique}

Velocity measurements are performed using a laser doppler velocimetry (LDV). A basic acquisition of 190.000 randomly sampled values of one velocity component at one point of the flow lasts about two minutes. Due to geometry constraints, we can measure the axial $V_{z}$ and tangential $V_{\theta}$ mean velocity components. From this raw data, one may compute the time-averaged flow at every point on a $11 * 15$ grid.

\section{Flow control parameters}

In the smooth case, the mean flow is mainly governed by three control parameters: the aspect ratio of the cavity $G$, the rotational Reynolds number $R e$ based on the cylinder radius and the ratio $\Gamma$ between the two rotation rates, defined as follows:

$$
0.01 \leq G=\frac{H}{R_{c}} \leq 1.8 \quad 2 \times 10^{5} \leq R e=\frac{\Omega_{1} R_{c}^{2}}{\nu} \leq 4 \times 10^{6} \quad-1 \leq \Gamma=-\frac{\Omega_{2}}{\Omega_{1}} \leq 0
$$

where $\nu$ is the kinematic viscosity of water. In the exact counter-rotating regime and in the rotor-stator configuration, the ratio $\Gamma$ is equal to -1 and 0 respectively. We define also the radial $r^{*}=r / R_{c}$ and axial $z^{*}=2 z / H$ coordinates. Thus, $r^{*}=0$ is obtained at the center of the disks and $r^{*}=1$ on the outer cylinder for $r=R_{c}$. In the same way, $z^{*}=-1$ on the lower disk 1 and $z^{*}=1$ on the upper disk 2 .

In the case of inertial stirring, the number of straight blades $n$ and their dimensionless height $h^{*}=h / R_{c}$ have also to be considered.

\section{STATISTICAL MODELING}

The predictions of the Reynolds Stress Model (RSM) used in the present work have already been validated in the rotor-stator configuration $(\Gamma=0)[32-35]$ for a wide range of aspect ratio $G$ and Reynolds number $R e$. It showed that this level of closure is adequate in such flow configurations, while the usual $k-\epsilon$ model, which is blind to any rotation effect presents serious deficiencies. Thus, the purpose of this paper relying on a well established turbulent model is to extend its application to new flow conditions and to get a better insight into the dynamics of the highly turbulent Von Kármán flow. 


\section{A. The differential Reynolds Stress Model (RSM)}

The flow studied here presents several complexities (high rotation rate, wall effects, transitional zone, shear layer), which are severe demands for turbulence modeling methods. Our approach is based on one-point statistical modeling using a low Reynolds number secondorder full stress transport closure derived from the Launder and Tselepidakis [36] model and sensitized to rotation effects [33]. This approach allows for a detailed description of near-wall turbulence and is free from any eddy viscosity hypothesis. The general equation for the Reynolds stress tensor $R_{i j}$ can be written:

$$
\frac{d R_{i j}}{d t}=P_{i j}+D_{i j}+\Phi_{i j}-\epsilon_{i j}+T_{i j}
$$

where $P_{i j}, D_{i j}, \Phi_{i j}, \epsilon_{i j}$, and $T_{i j}$ respectively denote the production, diffusion, pressure-strain correlation, dissipation and extra terms.

The diffusion term $D_{i j}$ is split into two parts: a turbulent diffusion $D_{i j}^{T}$, which is interpreted as the diffusion due to both velocity and pressure fluctuations [37] and a viscous diffusion $D_{i j}^{\nu}$, which cannot be neglected in the low Reynolds number region.

In a classical way, the pressure-strain correlation term $\Phi_{i j}$ can be decomposed in three parts: a slow nonlinear return to isotropy modeled as a quadratic development in the stress anisotropy tensor and damped near the wall, a linear rapid part which includes cubic terms and a wall correction applied to the linear part which is modeled using the Gibson and Launder hypothesis [38]. In this last term, the widely adopted length scale $k^{3 / 2} \varepsilon^{-1}$ is replaced by the length scale of the fluctuations normal to the wall.

The viscous dissipation tensor has been modeled in order to conform with the wall limits obtained from Taylor series expansions of the fluctuating velocities [39]. The extra term $T_{i j}$ accounts for implicit effects of the rotation on the turbulence field, it contains additional contributions in the pressure-strain correlation, a spectral jamming term, inhomogeneous effects and inverse flux due to rotation, which impedes the energy cascade [40]. A full description of the extra term $T_{i j}$ is given in [41].

The dissipation rate $\varepsilon$ equation to solve is the one proposed by Launder and Tselepidakis [36]. The turbulence kinetic energy $k$ equation which is redundant in a RSM model is still solved however, in order to get a more stable numerical convergence. It is verified that after convergence the turbulence kinetic energy $k$ is exactly equal to $0.5 R_{j j}$ within $0.05 \%$ at each 
grid point.

\section{B. Numerical method}

The computational procedure is based on a finite volume method using staggered grids for mean velocity components with axisymmetry hypothesis in the mean. The computer code is steady elliptic and the numerical solution proceeds iteratively. It has been verified that a $120 \times 120$ mesh in the $(r, z)$ frame is sufficient in smooth rotating disk cases to get grid-independent solutions. A refined mesh $160 \times 160$ is necessary to model flows with straight blades. It is to be compared to the $140 \times 80$ mesh used by Elena and Schiestel $[32,33]$ and Poncet et al. [34, 35] in rotor-stator systems. The calculation is initialized using realistic data fields, which satisfy the boundary conditions. About 20000 iterations (almost 20 hours on the bi-Opteron 18 nodes cluster of IRPHE) are necessary to obtain the numerical convergence of the calculation. The stress component equations are solved using matrix block tridiagonal solution to enhance stability using non staggered grids.

\section{Boundary conditions}

At the wall, all the variables are set to zero except for the tangential velocity $V_{\theta}$, which is set to $\Omega_{1} r$ on disk $1,-\Omega_{2} r$ on disk 2 and zero on the stationary cylinder. The usual value $\epsilon=\nu k_{, j} k_{, j} /(2 k)$ is imposed at the wall for the dissipation rate $\epsilon$ of the turbulence kinetic energy. At the periphery of the disks, for $R \leq r \leq R_{c}, V_{\theta}$ is supposed to vary linearly from zero on the stationary cylinder up to $\Omega_{1} R$ on disk 1 and $-\Omega_{2} R$ on disk 2 and the radial $V_{r}$ and axial $V_{z}$ velocity components are fixed to zero.

We can not implement real straight blades in our two-dimensional code. So we limit to modeling their most important effect, which is to increase the efficiency of the disks in forcing the flow. Thus, we add a volumic drag force $f$ in the equation of the tangential velocity component $V_{\theta}$. If we consider $n$ straight blades, the volumic drag force $f$ can be written as:

$$
f=\frac{n}{2 \pi r} F=\frac{n}{4 \pi r} \rho C_{D}\left|V_{r e l}\right| V_{r e l}
$$

where $F$ is the drag force of one blade, $\rho$ the fluid density, $C_{D}$ the dimensionless drag 
coefficient and $V_{r e l}=\Omega_{i} r-V_{\theta}$ the relative tangential velocity on disk $i=1,2$. The force is designed to make the fluid velocity closer to the local disk velocity near the disks. This form is close to the one proposed by Boroński [24] for spectral code. For curved blades, the same approach can be used: a volumic lift force can be added in the equation of the radial velocity component $V_{r}$. This will be the subject of a next study.

We can consider here that the height of the blades $\left(h^{*}=0.1\right.$ or 0.2$)$ is much larger than the boundary layer thickness $\delta / R_{c} \simeq R e^{-1 / 2} \leq 2 \times 10^{-3}$ (for $R e=2 \times 10^{5}$ ). In this case, Blevins [42] proposed some values for the drag coefficient (expected errors of $\pm 20 \%$ ) in an uniform flow. For a thin rectangular plate perpendicular to the mean flow, the value of $C_{D}$ is in the range $[1.05-1.9]$, depending on the size of the plate. As the flow is here not uniform along the blades and as the drag coefficient is supposed to decrease for increasing values of the Reynolds number, $C_{D}$ is expected to be lower than 2 depending on the flow and blade parameters. Some calculations have been performed for $\Gamma=-1, G=1.8, R e=2 \times 10^{5}$ and straight blades $\left(h^{*}=0.2, n=8\right)$ to study the influence of the trailing coefficient $C_{D}$. The differences on the extrema of the tangential velocity component are inferior to $0.5 \%$ for $C_{D}$ in the range $[0.1-2]$. Thus, we have chosen to fix the value of $C_{D}$ equal to 0.5.

The reader is thus referred to [32-35] for more details about the RSM model and the numerical method.

\section{SMOOTH DISK CASE: VISCOUS STIRRING}

In this section, we consider the turbulent flow between two counter-rotating flat smooth disks. Thus, we ensure a viscous stirring: the actuation is done by the setting in rotation of the smooth walls and the movement is communicated to the fluid by diffusion of the momentum through the boundary layers. We investigate the influence of the Reynolds number $R e$, the aspect ratio of the system $G$, and the ratio $\Gamma$ between the two rotation rates on the mean and turbulent fields.

\section{A. Flow structure in the exact counter-rotating regime}

The structure of the mean flow in the exact counter-rotating regime is henceforth globally well known: it can be decomposed into two toroidal cells in the tangential direction $\theta$ (not 
modelled here because of the axisymmetry hypothesis in the mean) and into two poloidal recirculations in the $(r, z)$ plane [28].

We focus here on the poloidal cells (fig.5a): the fluid at the top and the bottom of the cavity is forced into two opposite rotation speeds, and is then entrained by the disks. Consequently, a shear layer develops in the equatorial plane. This is perceptible in figure 2, which presents axial variations of the tangential velocity component for $\Gamma=-1, R e=6.28 \times 10^{5}$, $G=1.8$ at five radial locations in the range $r^{*}=0.346-0.865$. The radial and axial velocity components are not presented here because they are almost zero in the whole cavity both in the experiments and in the calculations. The tangential component is quite weak too except in the two very thin boundary layers, which develop on each disk and whose size is shown in figure 3 and close to the periphery, where the shear layer is observed. For $r^{*} \leq 0.476$ (fig.2a,b), the profile exhibits a Stewartson [2] flow structure: a quasi zero tangential velocity zone enclosed by two boundary layers on each disk. The flow in the boundary layers is characterized by a strong tangential velocity component (positive on disk 1 and negative on disk 2) and by a radial outward component not shown here. Towards the periphery (fig.2c-e), the flow gets of Batchelor type with five distinct zones: two boundary layers on the disks, a shear layer at mid-plane and two zones enclosed between the two. These last two zones are characterized by a weak but non zero tangential velocity component. The shear layer thickens when the local radius $r^{*}$ increases. Contrary to the laminar case reported by Kilic et al. [29], there is practically no radial inflow around $z^{*}=0$.

A good agreement between the numerical results and the experimental data is obtained even the values are quite weak. The RSM model catches the appearance and the thickening of the shear layer. On the other hand, the size of the LDV probe volume in the axial direction $(1 \mathrm{~mm})$ is not negligible compared to the boundary layer thickness. It is the main reason why the agreement between the numerical predictions and the measurements is less satisfactory in the boundary layers as it can be seen figure 2 .

The transition between the Stewartson and Batchelor flow structures can also be seen in figure 3 from the radial evolution of the boundary layer thickness $\delta$ for the same set of parameters. Very close to the rotation axis, the axial flow impinges the disks and creates very large boundary layers on both disks, whose size decreases with the local radius as expected [43]. The flow is then of Stewartson type. During the transition, $\delta$ increases as already observed by Poncet [35] for rotor-stator flows $(\Gamma=0)$. For $r^{*} \simeq 0.47$, the flow is clearly of 
Batchelor type and then, $\delta$ decreases towards the periphery of the cavity. It confirms the visualizations of the laminar flow between co- and counter-rotating disks $(-0.2 \leq \Gamma \leq 0.87)$ performed by Gauthier et al. [43]. They found indeed that the boundary layer thickness of the slower disk decreases for increasing values of the radial location $r^{*}$.

We investigate the influence of the Reynolds number on the mean flow. Figure 4 presents radial profiles of the tangential velocity component for $\Gamma=-1, G=1.8$ and four Reynolds numbers at different axial locations. The numerical predictions of the RSM model are compared to present LDV measurements and to the velocity measurements of Ravelet [21] for $R e \geq 10^{5}$. These data are also compared to the local disk 1 and disk 2 velocities, which are respectively $\Omega_{1} r$ and $-\Omega_{2} r$. The numerical data for $R e \geq 6.28 \times 10^{5}$ merge almost into a single fitting curve. It means that there is practically no effect of the Reynolds number on the mean field ever since the flow is turbulent. For $R e=2 \times 10^{5}$, a significant increase of the magnitude of $V_{\theta}$ is observed whatever the axial position, which is characteristic of the laminar regime. The critical Reynolds number for the transition from the laminar to the turbulent state is thus overestimated compared to the one obtained by Ravelet [21]: $R e=10^{5}$. Nevertheless, the present velocity measurements performed on the same experimental set-up as [21] confirm the numerical results. Compared to the previous measurements, an effect of $R e$ is observed on the radial profiles of $V_{\theta}$ at the periphery of the cavity. In fact, the critical Reynolds number for the laminar to turbulent state transition depends strongly on the boundary conditions and especially on the conditions imposed in the radial gap $0.925 \leq r^{*} \leq 1$. We recall that a linear profile is imposed in the numerical code for $V_{\theta}$, that does not take into account any recirculation zone and that could explain this difference. This tendency for relaminarization of the RSM model has already been noticed by Poncet et $a l .[34,35]$ in the rotor-stator configuration. As a conclusion, there is no significant effect of the Reynolds number on the mean flow for $R e \geq 10^{5}$, which confirms the results of Cadot et al. [16] and Ravelet [21].

Figure 5 presents the corresponding streamline patterns. The mean flow is divided into two symmetric poloidal cells, whose size is equal here to $0.5 \mathrm{H}$ along the axial direction and independent of the Reynolds number. In the radial direction, the diameter of the largest eddies observed is of the order of the disk radius $R$, showing this scale is the order of the energy scale injection. Experimentally, Ravelet [28] observed a weak dissymmetry of the flow in the $(r, z)$ plane, which disappears for increasing values of the Reynolds number. 
The influence of the aspect ratio of the cavity $G$ on the mean field has also been investigated for $0.01 \leq G \leq 1.8$ (fig.6) and a given Reynolds number $R e=1.3 \times 10^{6}$. Note that the radial and most of all the axial velocity components are quite weak compared to the tangential one and to the disk velocity. For $G=1.8$, the boundary layers are separated as already mentioned and the mean tangential velocity component is constant in the core of the flow. For $G=0.01$, the flow is of torsional Couette type with merged boundary layers as $V_{\theta}$ (fig.6a) varies linearly in the median region of the flow. This is to be compared to the value $G=0.012$ obtained in the rotor-stator configuration [35]. For intermediate values $G \simeq 0.4$, both boundary layers interact. The transition between the two main regimes is continuous and not clear from the $V_{\theta}$-profile. Nevertheless, if we consider the $V_{z}$-profile (fig.6c), we can clearly see that the axial velocity component is almost zero whatever the value of $G$, expect for $G=0.4$, where the fluid moves towards the upper and lower disks. The transition can also be characterized by considering the $V_{r}$-profiles (fig.6b), which exhibit the thinning of the boundary layers for increasing values of the aspect ratio.

\section{B. Flow structure for $-1 \leq \Gamma \leq 0$}

Another interesting feature in counter-rotating disk flows is the influence of the ratio $\Gamma$ between the two rotating disk speeds (fig.7). The Reynolds number and the aspect ratio of the cavity are respectively fixed to $R e=1.3 \times 10^{6}$ and $G=1.8$. We focus on the counterrotating disk case for which $-1 \leq \Gamma \leq 0$.

In the exact counter-rotating regime (fig.7a), the flow is symmetric and two cells with the same size $0.5 H$ coexist. For small rotating speed differences, the structure of the mean flow is strongly dominated by the faster disk (fig.7b). Varying the ratio $\Gamma$ displaces the shear layer towards the slower disk. The cell close to the lower disk invades almost the whole interdisk spacing for $\Gamma=-0.7$ (fig.7d). For $\Gamma=-0.2$ (fig.7e), the flow structure resembles the one observed in the rotor-stator configuration [35] with streamline patterns parallel to the rotating axis. This transition between the two cell and the one cell regimes can be seen also from figure 8. It presents the evolution with $\Gamma$ of the dimensionless size $S_{c} / H$ of the smallest cell (along the upper disk) in the axial direction defined in figure 7b. In the smooth disk case, we notice that $S_{c}$ decreases rapidly for decreasing values of $|\Gamma|$ in the range $-1 \leq \Gamma \leq-0.8$ (see also fig.7a-c) following $S_{c} / H \propto-2.2 \Gamma$. For smallest values 
of $|\Gamma|$, the cell is reduced to a very thin region attached to the upper disk (fig.7d), which disappears progressively along the external cylinder and so $S_{c}$ tends to zero.

In figure 8, our results are compared to the ones obtained by Kilic et al. [29] and Gan et al. [31], who performed calculations for $-1 \leq \Gamma \leq 0$ and $G=0.12$ using a classical $k-\epsilon$ turbulence model. Considering that the $k-\epsilon$ prediction of these authors is in relatively good agreement with the smooth disk case, the comparison given in figure 8 may be meaningful to get an idea of the effect of aspect ratio. For $R e=10^{5}$, Kilic et al. [29] found that the evolution of $S_{c}$ against $\Gamma$ is non monotonous. It decreases more slowly from $\Gamma=-1$ to $\Gamma=-0.2$ than in our case. It is a combined effect of both the Reynolds number and the aspect ratio of the cavity. For $\Gamma=-0.4$, they observed a double transition: from laminar to turbulent flow and from Batchelor to Stewartson type of flow. The decrease of $S_{c}$ is much faster with $\Gamma$ in the laminar case [29]. For $R e=1.25 \times 10^{6}$, Gan et al. [31] obtained streamline patterns different from the ones shown in figure 7 for $\Gamma=[-0.8 ;-0.2]$ essentially because of the small value of $G$. A large cell along the slower disk is still observed for $\Gamma=-0.4$. This cell is trapped by the main flow due to the faster disk in the zone $0.3 \leq r^{*} \leq 0.45$.

\section{Turbulence field in the exact counter-rotating regime}

As already mentioned above, the influences of both the Reynolds number and the aspect ratio are relatively weak (compared to the effect of the ratio $\Gamma$ between the rotation rates). In the following, we focus on the exact counter-rotating regime $\Gamma=-1$ and $R e$ and $G$ are fixed respectively to $R e=6.28 \times 10^{5}$ and $G=1.8$.

Figure 9 presents the axial profiles of the six components of the Reynolds stress tensor. These components are normalized by the local disk 1 velocity $\Omega_{1} r$. For example, $R_{r r}^{*}$ is defined as: $R_{r r}^{*}=\overline{v_{r}^{\prime 2}} /\left(\Omega_{1} r\right)^{2}$. As in all rotating disk problems [34], turbulence is mainly concentrated in the boundary layers with the same turbulence levels in the upper and lower disk boundary layers. The main difference with the rotor-stator configuration is that turbulence is also generated in the median region of the interdisk spacing and is due to the shear, stretched by the recirculations. The Von Kármán arrangement is indeed known to produce an intense turbulence in a compact region of space [14]. The magnitudes of the three normal components (in principal axes) are almost the same in the equatorial plane. It means that turbulence is quasi isotropic in that region. The cross components are quite weak except for 
the $R_{r \theta}^{*}$ component, which behaves like the normal components with a bump at mid-plane.

As expected, the maximum of the turbulence Reynolds number $R e_{t}=k^{2} /(\nu \epsilon)=5836$ is located in the shear layer close to the periphery of the cavity, where the highest values of the local Reynolds number $R e_{r}=\Omega_{1} r^{2} / \nu$ are obtained (fig.10a). This maximum is to be compared to the maximum value $R e_{t} \simeq 500$ obtained by Poncet [35] for $\Gamma=0$ and $R e \simeq 10^{6}$, which indicates the high turbulence level in that region.

Figure 10b shows the anisotropy invariant map for the Reynolds stress tensor in the whole interdisk spacing at $r^{*}=0.51$. The second $A_{2}$ and third $A_{3}$ invariants of the anisotropy tensor $a_{i j}$ of the second moments of the fluctuations are defined as: $A_{2}=a_{i j} a_{j i}$ and $A_{3}=a_{i j} a_{j k} a_{k i}$ [44], where $a_{i j}=R_{i j} / k-\frac{2}{3} \delta_{i j}\left(\delta_{i j}\right.$ the Kronecker symbol). The results of the RSM model satisfy the realizability diagram of Lumley [44]. Very close to the disks, the turbulence tends to follow the two-component behavior as the wall normal fluctuations are damped more effectively than fluctuations parallel to the disk. Outside the boundary layers and especially in the shear layer, the turbulence is fairly close to the isotropic case $\left(A_{2}=A_{3}=0\right)$, which confirms the results observed from figure 9 . Note that very close to the mid-plane, the flow tends to the axisymmetric limit.

\section{BLADED DISK CASE: INERTIAL STIRRING}

To increase the efficiency of the disks in forcing the flow, we used $n$ blades of height $h^{*}$ mounted on both disks. The stirring is called inertial because the fluid is set into motion thanks to areas of forcing perpendicular to the motion itself. In that case, Ravelet [21] showed that all mean and turbulent quantities are independent of the Reynolds number in the range $R e=\left[10^{5}, 2 \times 10^{6}\right]$. Thus, we have chosen to fix the values of $R e \simeq 2 \times 10^{5}$ and $G=1.8$. In that case, the boundary layers are separated and the flow is found to be highly turbulent. Moreover, direct comparisons with the experiments of Ravelet [21] can be performed. The purpose of this section is to propose an efficient way to model the effect of straight blades on both the mean and turbulent fields. 


\section{A. Flow structure in the exact counter-rotating regime}

In the bladed disk case, the flow structure is completely different from the smooth disk case, where the velocity gradient are located in the boundary layers along the disks and decrease when the Reynolds number increases. For an inertially driven flow, the mean flow does not present any appreciable velocity gradient in the vicinity of the blades (fig.11) and the gradients are distributed in the median region of the flow. The mean flow is divided into three main regions: a shear layer at mid-plane and two fluid regions close to each bladed disks. The intensity of the shear at mid-plane is increased compared to the viscous stirring case. This shear is due to the two recirculation cells. It induces a strong radial inflow $\left(V_{r}<0\right)$ around $z^{*}=0$ and two opposite axial flows towards the disks. The magnitude of the mean axial and radial velocity components increase from the periphery (fig.11c) to the rotation axis (fig.11a). From the disk to the top of the blades, the tangential fluid velocity is fairly close to the local disk velocity. Moreover, a strong radial outflow is created along the bladed disks and goes with the impellers. At the top of the blades, there is a strong decrease of $\left|V_{\theta}\right|$ interpreted as the wake of the blades. There is a very good agreement between the numerical predictions and the velocity measurements concerning the $V_{\theta}$-profiles. A small difference is observed in the shear layer, where the RSM model predicts a thinner layer than the one measured by Ravelet [21]. This last author observed, for the same set of parameters, high energy levels for frequencies inferior to the injection frequency. This contribution is attributed to the appearance of strong coherent structures in the shear layer not observed in the smooth disk case and which may explain the weak discrepancies obtained.

\section{B. Flow structure for $-1 \leq \Gamma \leq 0$}

We perform the same analysis as in the smooth disk case by varying the ratio $\Gamma$ between the two rotating disk speeds. Figure 8 presents comparisons between the smooth and bladed disk cases concerning the size $S_{c}$ of the cell along the slowest disk for $-1 \leq \Gamma \leq 0$. The same behavior is obtained but the transition between the two cell and the one cell structures $\left(S_{c} \rightarrow\right.$ 0 ) is slightly delayed. It occurs in the inertial stirring case for $\Gamma \simeq-0.65$, which is close to the experimental value obtained by Cadot and Le Maître [19] in the same configuration $\Gamma=-0.69$ and the analytical one obtained by Dijkstra and Van Heijst [9] for $R e \rightarrow 0$ in 
the smooth disk case $\Gamma=-2 / 3$. The measurements of Ravelet [21] reveal a transition for $\Gamma=-0.78$. It confirms the similitude observed by [19] between the smooth disk flow with a large viscosity and the mean flow in the inertial stirring case.

The transition from the two cell to the one cell structures can be seen also from figure 12. Compared to the smooth disk case, the cell along the slowest disk is larger for $\Gamma=-0.8$ (fig.12b). For $\Gamma=-0.7$, only a small recirculation subsists along the upper disk and completely disappears for $\Gamma=-0.6$. For $\Gamma \geq-0.6$, the same pattern is observed with streamlines parallel to the rotation axis.

\section{Turbulence field in the exact counter-rotating regime}

To enable direct comparisons with the viscous stirring case, figure 13 presents the axial profiles of the six components of the Reynolds stress tensor at the same radius $r^{*}=0.81$ and for the same values of $G$ and $\Gamma$. The main difference between the smooth and the bladed disk configurations is that, in the latter case, the turbulence intensities vanish towards the disks. Apart from that, turbulence is also mostly generated at mid-plane because of the shear stretched by the recirculations. The blades induce a much stronger shear zone in the equatorial plane compared to the smooth disk case as already seen from the mean velocity profiles (fig.11). Thus, the turbulence levels, regarding the normal Reynolds stress components (fig.13a), are almost 20 times larger than for viscous stirring and quite comparable to the mean fluid velocity. It confirms the previous measurements of Cadot et al. [16] in steady regimes of turbulence in the Von Kármán geometry. They found that the fluid velocity fluctuations are close to the fluid mean velocity and 6 times larger in the bladed disk case than in the smooth disk case. In the present study, the $R_{r r}^{*}$ component is much weaker than the two other normal components, which indicates the turbulence anisotropy in the core of the flow. The cross components are also stronger than in the smooth disk case. The level of the $R_{r \theta}^{*}$ component (fig.13b) is of the order of $R_{r r}^{*}$. Note that the maximum of the $R_{\theta \theta}^{* 1 / 2}$ component obtained at mid-plane $\left(z^{*}=0\right)$ using the RSM model is in excellent agreement with the asymptotic value measured by Ravelet [21] for $R e \geq 10^{4}$ (relative error inferior to $0.1 \%$ ). Nevertheless, one must remark that only a single measurement point is available, and consequently it is hard to derive definite conclusions. Another point is that the periodic unsteadiness introduced by the blades is not exactly accounted for in the calculation and 
may explain small discrepancies in the mean velocity profiles.

To study the influence of the number $n$ of blades and their height $h^{*}$ on the turbulent field, figure 14 shows radial profiles of the turbulence kinetic energy $k^{*}$ normalized by $\left(\Omega_{1} R_{c}\right)^{2}$ for various impeller configurations. These profiles are plotted at mid-plane where the maximum of $k^{*}$ prevails. As expected, $k^{*}$ increases towards the periphery of the cavity, it means for increasing local Reynolds number. Then, $k^{*}$ decreases for radial locations in the gap between the disks and the external cylinder. We can first notice the very weak level of turbulence kinetic energy in the smooth disk case compared to the other bladed disk cases. Secondly, the influence of the blade number $n$ is quite weak for $n=4,8$ or 16 . Only very close to the rotation axis, we can notice a different behavior in the configuration with 16 blades. Nevertheless, in the whole flow, four blades seem to be sufficient to force the flow. On the other hand, the blade height $h$ plays a more important role. The $k^{*}$ level is twice higher when the blades are twice higher too.

It is now established that all mean and turbulent quantities are independent of the Reynolds number in the range $R e=\left[10^{5}, 2 \times 10^{6}\right]$. The turbulent dissipation is indeed much stronger than the dissipation due to the boundary layers and hides the dependence on Re. All these results can thus be extended to higher Reynolds numbers.

\section{CONCLUSION}

We have performed some comparisons between numerical predictions using a RSM model and velocity measurements considering the turbulent flow between two flat or bladed counterrotating disks. This configuration known as the Von Kármán geometry is used to produce an intense turbulence in a compact region of space.

For viscous stirring, the flow is of Stewartson type close to the rotation axis and so exhibits three distinct regions: two boundary layers and one shear layer at mid-plane. When one approaches the periphery of the cavity, for $r^{*} \simeq 0.476$, the flow gets of Batchelor type. Turbulence is mainly concentrated in the boundary layers and in the transitional shear layer, where turbulence is almost isotropic. Turbulence intensities increase towards the outer cylinder. When one decreases the aspect ratio of the cavity until $G \leq 0.4$, the boundary layers mixed and the flow is then of torsional Couette type for lower values of $G$. In the case of inertial stirring, the impellers are more efficient to force the flow. Thus, the transitional 
shear layer intensifies. Turbulence is so mainly concentrated around $z^{*}=0$ and vanish towards the disks. The turbulence intensities are almost 20 times larger than in the flat disk case. The height of the blades is found to be the preponderant parameter to increase the turbulence intensities more than the number of blades. In the flat and bladed disk cases, we have numerically verified the statement of Cadot et al. [16]: "smooth or rough, the efficiency of a given type of stirrer to set the bulk of the fluid in motion is independent of the Reynolds number". Moreover, we have characterized the transition between the two cell and the one cell regimes. For inertial stirring, it occurs for $\Gamma \simeq-0.65$ close to the values obtained by $[9,19]$.

The agreement between the numerical predictions and the LDV measurements is very satisfactory in both cases. For the first time, an easy and efficient way to model the main effect of straight blades has been proposed. Further experimental works are now required to provide more comparisons for the turbulent fields but also some calculations for curved blades.

[1] G.K. Batchelor. Note on a class of solutions of the Navier-Stokes equations representing steady rotationally-symmetric flow. Quat. J. Mech. and Appl. Math., 4(1):29-41, 1951.

[2] K. Stewartson. On the flow between two rotating coaxial disks. Proc. Camb. Phil. Soc., 49:333-341, 1953.

[3] T. Von Kármán. Uber laminare und turbulente Reibung. Z. Angew. Math. Mech., 1:233-252, 1921.

[4] G.N. Lance and M.H. Rogers. The axially symetric flow of a viscous fluid between two infinite rotating disks. Proc. R. Soc. London A, 266:109-121, 1962.

[5] J.B. McLeod and S.V. Parter. On the flow between two counter-rotating infinite plane disks. Arch. Ration. Mech. Anal., 54:301-327, 1974.

[6] H.J. Pesch and P. Rentrop. Numerical solution of the flow between two counter-rotating infinite plane disks by multiple shooting. ZAMM, 58:23-28, 1978.

[7] H.O. Kreiss and S.V. Parter. On the swirling flow between rotating coaxial disks: existence and uniqueness. Commun. Pure Appl.Math., 36:55-84, 1983.

[8] C.E. Pearson. Numerical solutions for time-dependent viscous flow between rotating coaxial 
disks. J. Fluid Mech., 21(4):623-633, 1965.

[9] D. Dijkstra and G.J.F. Van Heijst. The flow between two finite rotating disks enclosed by a cylinder. J. Fluid. Mech., 128:123-154, 1983.

[10] C. Yang and S. Liao. On the explicit, purely analytic solution of Von Kármán swirling viscous flow. Communications in Nonlinear Science \& Numerical Simulation, 11:83-93, 2006.

[11] M. Holodniok, M. Kubicek, and V. Hlavacek. Computation of the flow between two rotating coaxial disks: multiplicity of steady-state solutions. J. Fluid. Mech., 108:227-240, 1981.

[12] P.J. Zandbergen and D. Dijkstra. Von Kármán swirling flows. Ann. Rev. Fluid Mech., 19:465$491,1987$.

[13] S. Fauve, C. Laroche, and B. Castaing. Pressure fluctuations in swirling turbulent flows. J. Phys. II France, 3:271-278, 1993.

[14] J. Maurer, P. Tabeling, and G. Zocchi. Statistics of turbulence between two counterrotating disks in low-temperature helium gas. Europhys. Lett., 26(1):31-36, 1994.

[15] N. Mordant, J.-F. Pinton, and F. Chillà. Characterization of turbulence in a closed flow. J. Phys. II France, 7:1729-1742, 1997.

[16] O. Cadot, Y. Couder, A. Daerr, S. Douady, and A. Tsinober. Energy injection in closed turbulent flows: stirring through boundary layers versus inertial stirring. Phys. Rev. E, 56(1):427433, 1997.

[17] J.-F. Pinton, P.C.W. Holdsworth, and R. Labbé. Power fluctuations in a closed turbulent shear flow. Phys. Rev. E, 60(3):2452-2455, 1999.

[18] L. Marié and F. Daviaud. Experimental measurement of the scale-by-scale momentum transport budget in a turbulent shear flow. Phys. Fluids, 16(2):457-461, 2004.

[19] O. Cadot and O. Le Maître. The turbulent flow between two rotating stirrers: similarity laws and transitions for the driving torques fluctuations. Eur. J. Mech. B/Fluids, in press.

[20] F. Ravelet, L. Marié, A. Chiffaudel, and F. Daviaud. Multistability and memory effect in a highly turbulent flow: experimental evidence for a global bifurcation. Phys. Rev. Letters, 93(16), 2004.

[21] F. Ravelet. Bifurcations globales hydrodynamiques et magnétohydrodynamiques dans un écoulement de Von Kármán turbulent. PhD thesis, Groupe Instabilités et Turbulence, Service de Physique de l'État Condensé, CEA Saclay, 2005.

[22] R. Monchaux, F. Ravelet, B. Dubrulle, A. Chiffaudel, and F. Daviaud. Properties of steady 
states in turbulent axisymmetric flows. Phys. Rev. Lett., 96(12):124502, 2006.

[23] N. Leprovost, B. Dubrulle, and P.-H. Chavanis. Dynamics and thermodynamics of axisymmetric flows: Theory. Phys. Rev. E, 73(046308), 2006.

[24] P. Boroński. Méthode des potentiels polö̈dal-toroïdal appliquée à l'écoulement de Von Kármán en cylindre fini. PhD thesis, École Polytechnique, 2005.

[25] M. Bourgoin, L. Marié, F. Pétrélis, C. Gasquet, A. Guigon, J.-B. Luciani, M. Moulin, F. Namer, J. Burguete, A. Chiffaudel, F. Daviaud, S. Fauve, P. Odier, and J.-F. Pinton. Magnetohydrodynamics measurements in the Von Kármán sodium experiment. Phys. Fluids, 14(9):3046-3058, 2002.

[26] R. Volk, P. Odier, and J.-F. Pinton. Fluctuation of magnetic induction in Von Kármán swirling flows. Phys. Fluids, 18(085105), 2006.

[27] M. Bourgoin, P. Odier, J.-F. Pinton, and Y. Ricard. An iterative study of time independent induction effects in magnetohydrodynamics. Phys. Fluids, 16(7):2529-2547, 2004.

[28] F. Ravelet, A. Chiffaudel, F. Daviaud, and J. Léorat. Toward an experimental Von Kármán dynamo: numerical studies for an optimized design. Phys. Fluids, 17(11):117104, 2005.

[29] M. Kilic, X. Gan, and J.M. Owen. Transitional flow between contra-rotating disks. J. Fluid Mech., 281:119-135, 1994.

[30] B.E. Launder and B.I. Sharma. Application of the energy dissipation model of turbulence to the calculation of flow near a spinning disc. Lett. Heat Mass Transfer, 1:131-138, 1974.

[31] X. Gan, M. Kilic, and J.M. Owen. Superposed flow between two discs contrarotating at differential speeds. Int. J. Heat Fluid Flow, 15(6):438-446, 1994.

[32] L. Elena. Modélisation de la turbulence inhomogène en présence de rotation. PhD thesis, Université Aix-Marseille I-II, 1994.

[33] L. Elena and R. Schiestel. Turbulence modeling of rotating confined flows. Int. J. Heat Fluid Flow, 17:283-289, 1996.

[34] S. Poncet, M.P. Chauve, and R. Schiestel. Batchelor versus Stewartson flow structures in a rotor-stator cavity with throughflow. Phys. Fluids, 17(7), 2005.

[35] S. Poncet. Écoulements de type rotor-stator soumis à un flux axial: de Batchelor à Stewartson. PhD thesis, Université de Provence, 2005.

[36] B.E. Launder and D.P. Tselepidakis. Application of a new second-moment closure to turbulent channel flow rotating in orthogonal mode. Int. J. Heat Fluid Flow, 15(1):2-10, 1994. 
[37] B.J. Daly and F.H. Harlow. Transport equation for turbulence. Phys. Fluids A, 13(11):2634$2649,1970$.

[38] M. Gibson and B.E. Launder. Ground effects on pressure fluctuations in the atmospheric boundary layer. J. Fluid. Mech., 86(3):491-511, 1978.

[39] B.E. Launder and W.C. Reynolds. Asymptotic near-wall stress dissipation rates in a turbulent flow. Phys. Fluids A, 26(5):1157-1158, 1983.

[40] C. Cambon and L. Jacquin. Spectral approach to non-isotropic turbulence subjected to rotation. J. Fluid Mech., 202(1), 1989.

[41] R. Schiestel and Elena. Modeling of anisotropic turbulence in rapid rotation. Aerospace Science and Technology, 7:441-451, 1997.

[42] R.D. Blevins. Applied fluid dynamics handbook. Ed. Van Nostrand Reinhold Company Inc., New-York, 1984.

[43] G. Gauthier, P. Gondret, F. Moisy, and M. Rabaud. Instabilities in the flow between co- and counter-rotating disks. J. Fluid Mech., 473:1-21, 2002.

[44] J.L. Lumley. Computational modeling of turbulent flows. Adv. Appl. Mech., 18:123-176, 1978. 
- Fig.1: Sketches of the cavity with (a) relevant notation in the smooth disk case and (b) straight blades.

- Fig.2: Axial profiles of the tangential velocity component for $\Gamma=-1, R e=6.28 \times 10^{5}$ and $G=1.8$ at five radial locations: (a) $r^{*}=0.346$, (b) $r^{*}=0.476$, (c) $r^{*}=0.605$, (d) $r^{*}=0.735$, (e) $r^{*}=0.865$. Comparisons between the numerical results $(-)$ and the experimental data (o) in the smooth disk case.

- Fig.3: Radial evolution of the boundary layer thickness $\delta / H$ in the smooth disk case for $\Gamma=-1, R e=6.28 \times 10^{5}$ and $G=1.8$ (symbols: RSM, line: polynomial interpolation).

- Fig.4: Radial profiles of the tangential velocity component in the smooth disk case for $\Gamma=-1, G=1.8$ and four Reynolds numbers at different axial locations: (a) $z^{*}=0.91$, (b) $z^{*}=0.59$, (c) $z^{*}=0.02$, (d) $z^{*}=-0.59$, (e) $z^{*}=-0.92$.

- Fig.5: Computed streamlines between smooth disks for $\Gamma=-1$ and $G=1.8$ : (a) $R e=2 \times 10^{5}$; (b) $R e=7.8 \times 10^{5}$; (c) $R e=1.3 \times 10^{6}$; (d) $R e=4 \times 10^{6}$.

- Fig.6: Axial profiles of the mean velocity components at $r^{*}=0.81$ for $\Gamma=-1$, $R e=1.3 \times 10^{6}$ and four values of $G$ in the smooth disk case (RSM).

- Fig.7: Computed streamlines between smooth disks for $R e=1.3 \times 10^{6}$ and $G=1.8$ : (a) $\Gamma=-1$; (b) $\Gamma=-0.9$; (c) $\Gamma=-0.8$; (d) $\Gamma=-0.7$; (e) $\Gamma=-0.2$.

- Fig.8: Size $S_{c} / H$ of the smallest cell against $\Gamma$ for $G=1.8$ (RSM). Comparison between $(-)$ the smooth disk case $\left(R e=1.3 \times 10^{6}\right),(--)$ the bladed disk case $\left(R e=2 \times 10^{5}\right)$ and previous numerical results of (०) Kilic et al. [29] and ( $\diamond)$ Gan et al. [31] in the smooth disk case.

- Fig.9: Axial profiles of the six Reynolds stress tensor components at $r^{*}=0.81$ for $\Gamma=-1, G=1.8$ and $R e=6.28 \times 10^{5}$ in the smooth disk case (RSM).

- Fig.10: $\Gamma=-1, G=1.8$ and $R e=6.28 \times 10^{5}$ in the smooth disk case (RSM): (a) Iso-turbulence Reynolds number $R e_{t}=k^{2} /(\nu \epsilon)$ - (b) Anisotropy invariant map at $r^{*}=0.51:(\times)-1 \leq z^{*} \leq 0,(\square) 0 \leq z^{*} \leq 1$.

- Fig.11: Axial profiles of the mean velocity components for $\Gamma=-1, R e=2 \times 10^{5}$, $G=1.8$ and straight blades $\left(n=8, h^{*}=0.2\right)$ at three radial locations: (a) $r^{*}=0.4$, 
(b) $r^{*}=0.5$, (c) $r^{*}=0.6$. Comparisons between the predictions of the RSM model (lines) and the LDV measurements of Ravelet [21] (o).

- Fig.12: Computed streamlines for $R e=2 \times 10^{5}, G=1.8$ and straight blades $(n=8$, $h^{*}=0.2$ ): (a) $\Gamma=-1$; (b) $\Gamma=-0.9$; (c) $\Gamma=-0.8$; (d) $\Gamma=-0.7$; (e) $\Gamma=-0.6$.

- Fig.13: Axial profiles of the six Reynolds stress tensor components at $r^{*}=0.81$ for $\Gamma=-1, G=1.8, R e=2 \times 10^{5}$ and straight blades $\left(n=8, h^{*}=0.2\right)(\mathrm{RSM})$. (०) LDV data of Ravelet [21] for $R_{\theta \theta}^{* 1 / 2}$.

- Fig.14: Radial profiles of the turbulence kinetic energy $k^{*}=k /\left(\Omega_{1} R_{c}\right)^{2}$ at $z^{*}=0$ for $\Gamma=-1, G=1.8, R e=2 \times 10^{5}$ and different bladed disk configurations - comparison with the smooth disk case $\left(R e=6.28 \times 10^{5}\right)(\mathrm{RSM})$. 


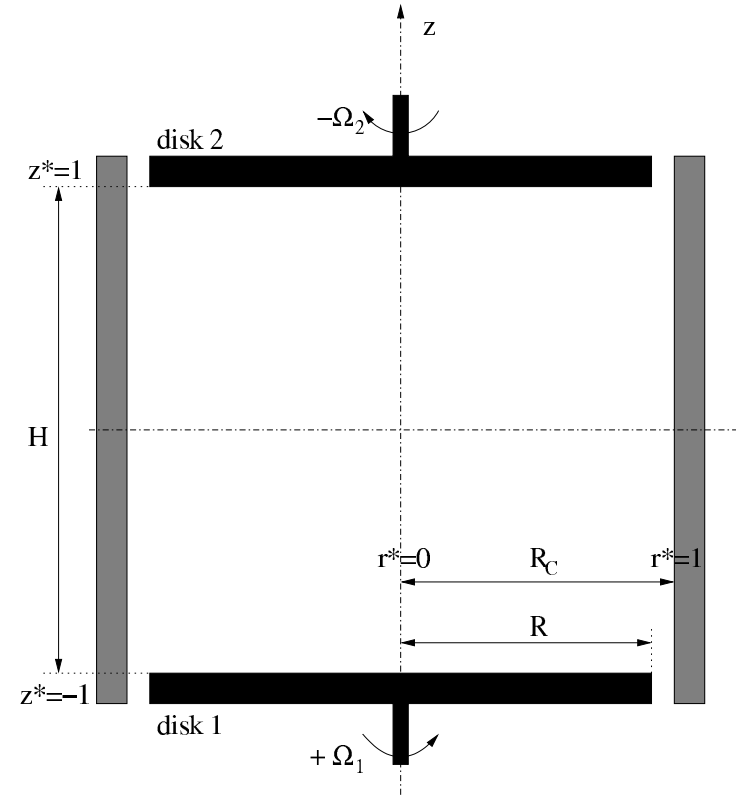

(a)

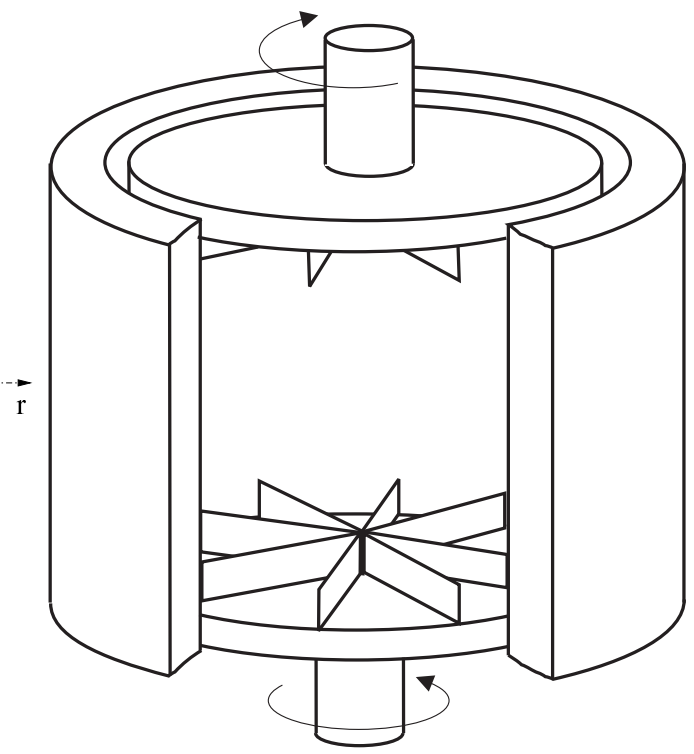

(b)

Figure 1: Poncet et al., submitted to Int. J. Heat Fluid Flow. 
(a)

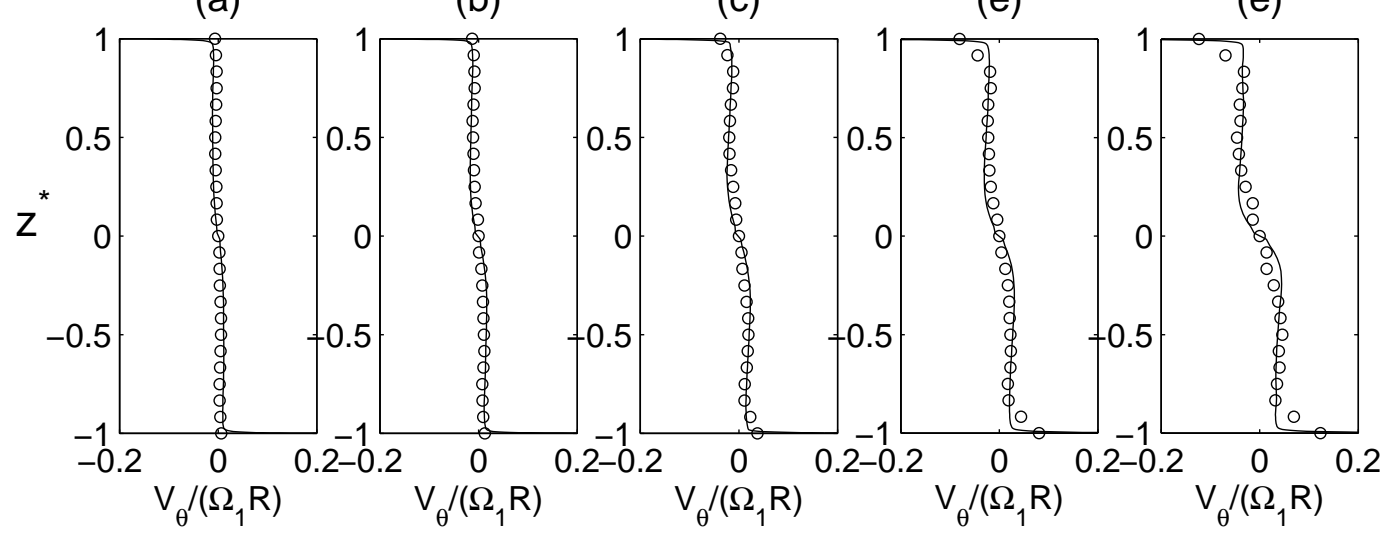

Figure 2: Poncet et al., submitted to Int. J. Heat Fluid Flow. 


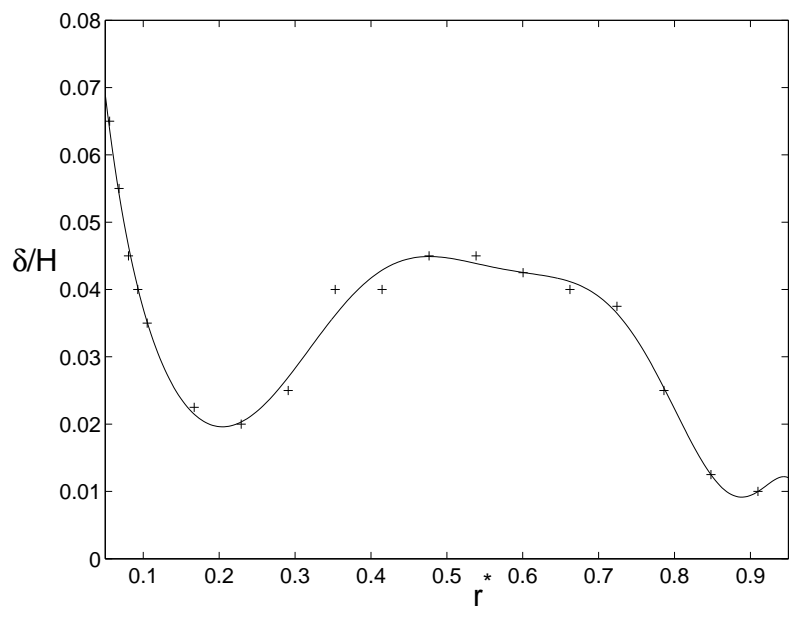

Figure 3: Poncet et al., submitted to Int. J. Heat Fluid Flow. 
(a)

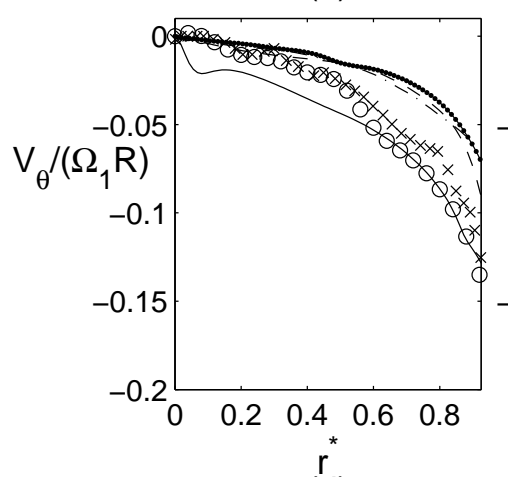

(d)

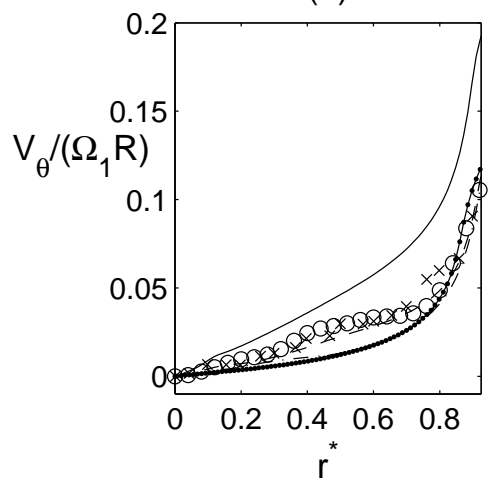

(b)

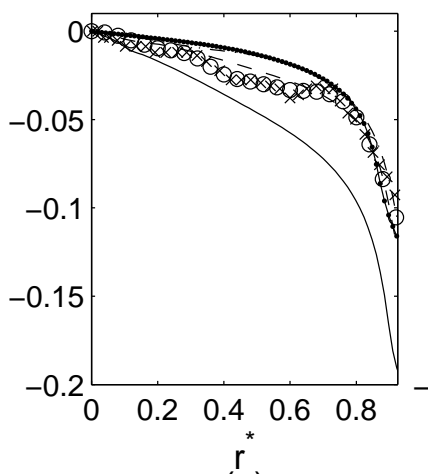

(e)

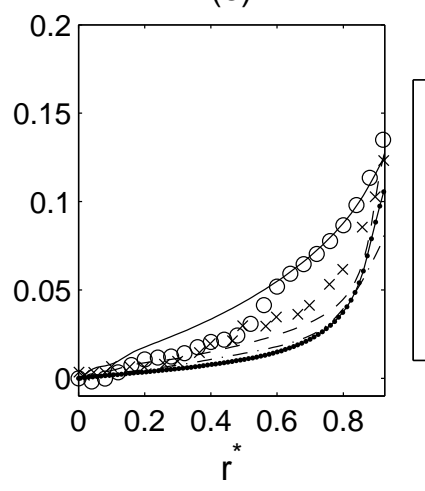

(c)

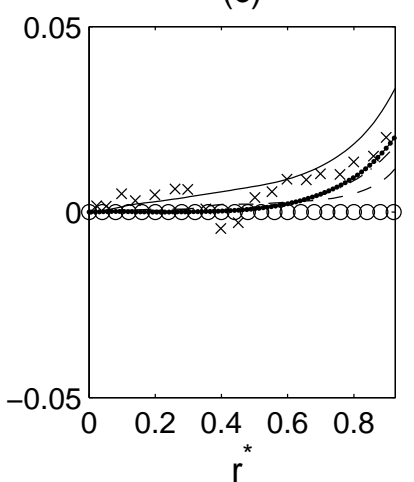

$\mathrm{Re}=2 \times 10^{5}$ (RSM)

$\mathrm{Re}=6.28 \times 10^{5}(\mathrm{RSM})$

- $R e=6.28 \times 10^{5}$ (LDV)

$\mathrm{Re}=1.3 \times 10^{6}(\mathrm{RSM})$

$\mathrm{Re}=4 \times 10^{6}$ (RSM)

$\mathrm{Re} \geq 10^{5}$ (Ravelet, 2005)

Figure 4: Poncet et al., submitted to Int. J. Heat Fluid Flow. 
(a)

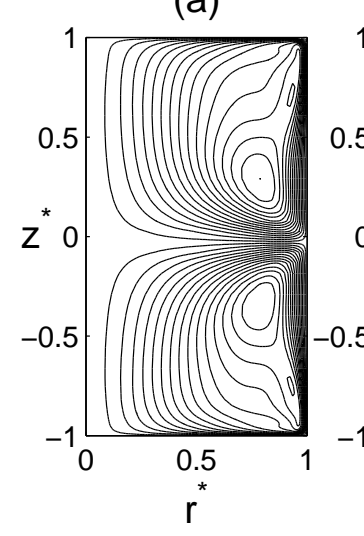

(b)

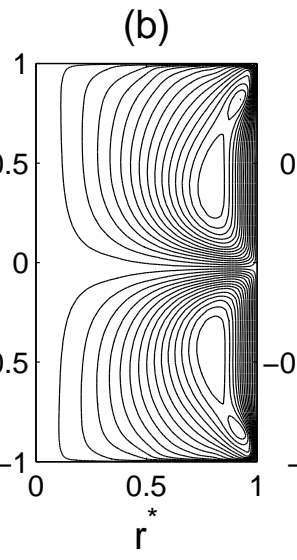

(c)

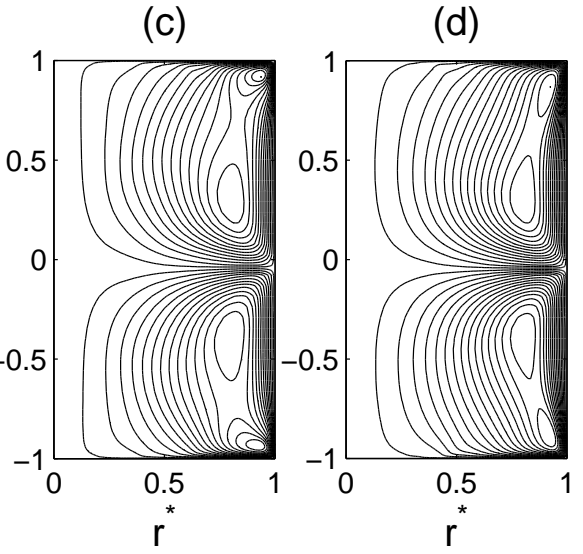

Figure 5: Poncet et al., submitted to Int. J. Heat Fluid Flow. 

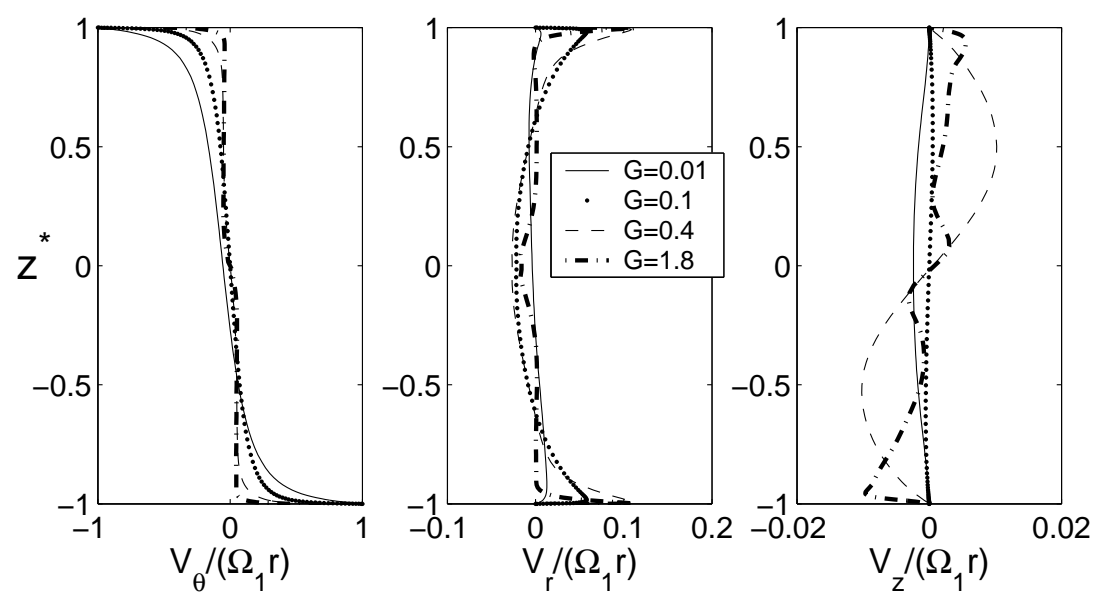

Figure 6: Poncet et al., submitted to Int. J. Heat Fluid Flow. 
(a)

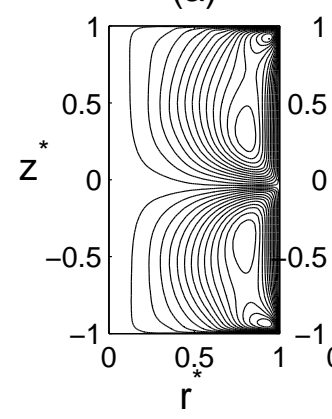

(b)

(c)

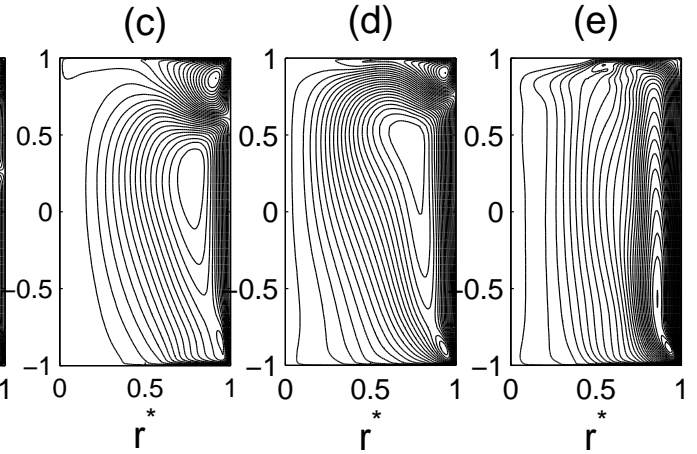

Figure 7: Poncet et al., submitted to Int. J. Heat Fluid Flow. 


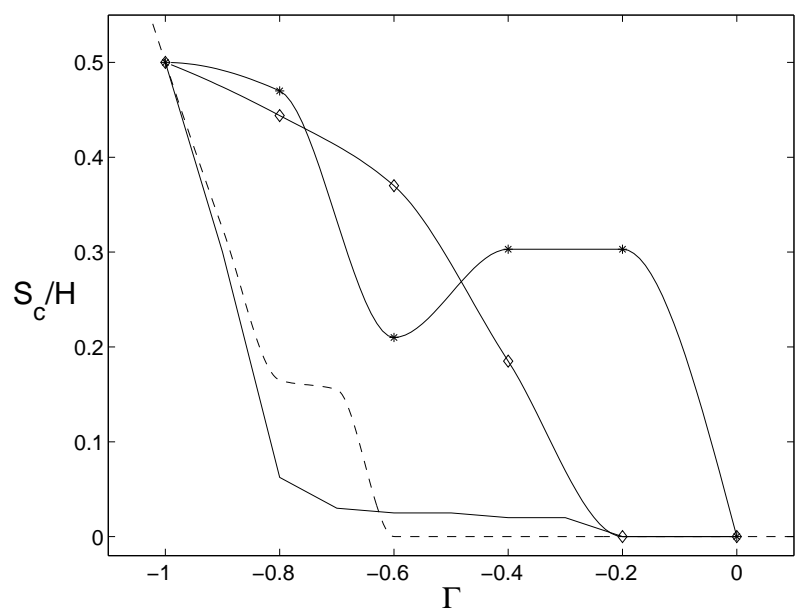

Figure 8: Poncet et al., submitted to Int. J. Heat Fluid Flow. 

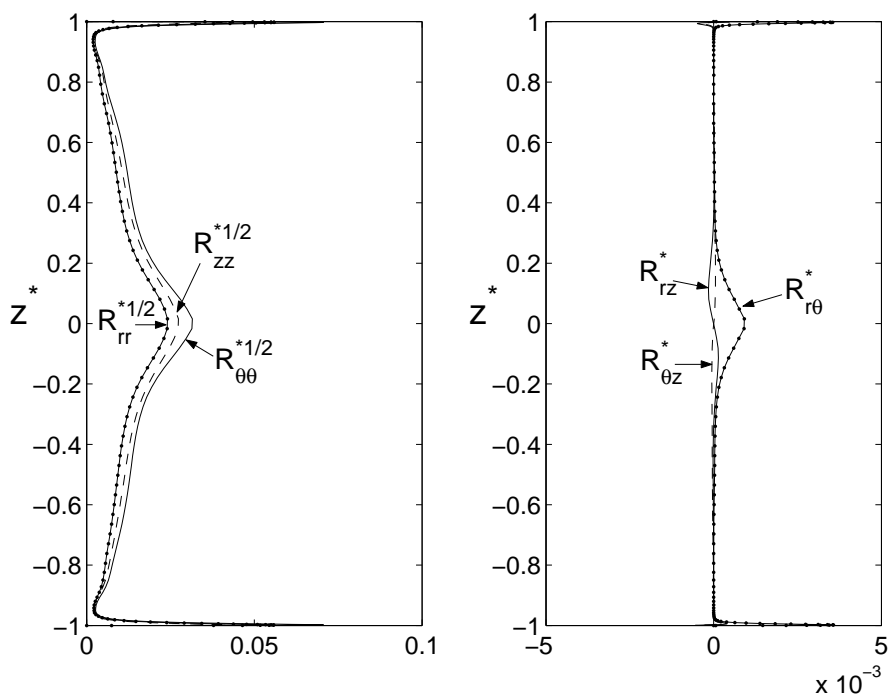

Figure 9: Poncet et al., submitted to Int. J. Heat Fluid Flow. 


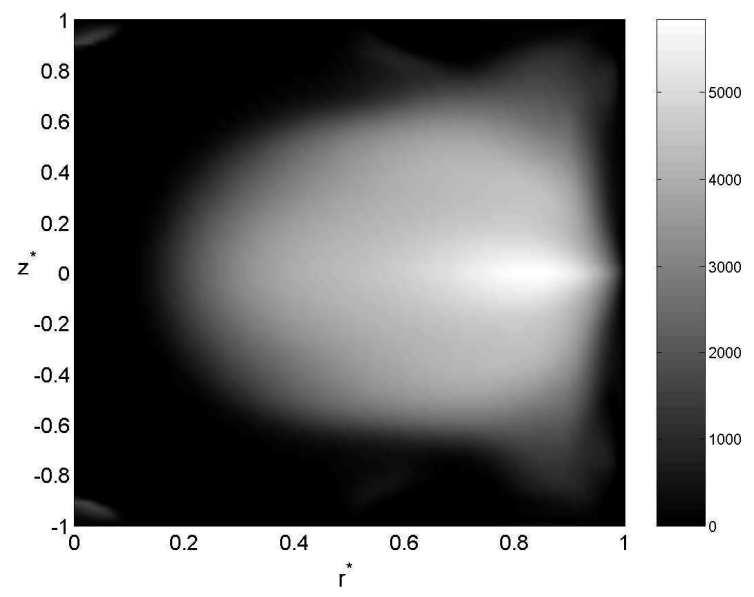

(a)

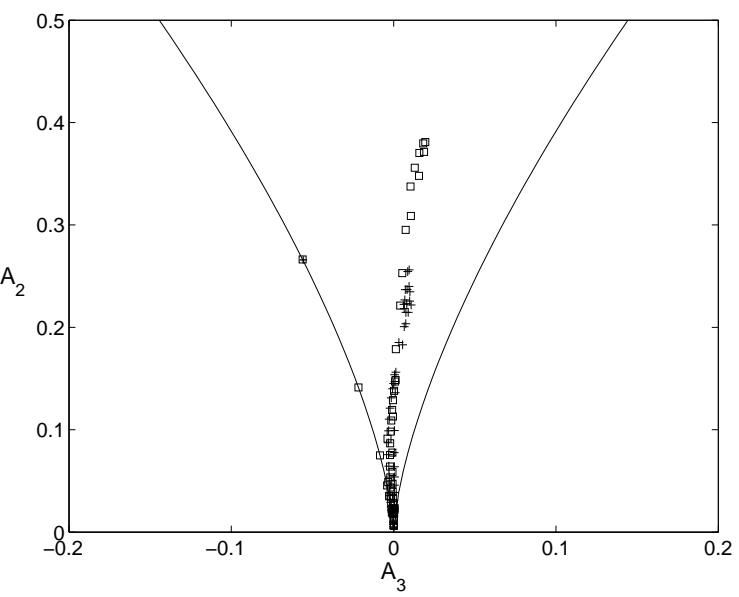

(b)

Figure 10: Poncet et al., submitted to Int. J. Heat Fluid Flow. 
(a)
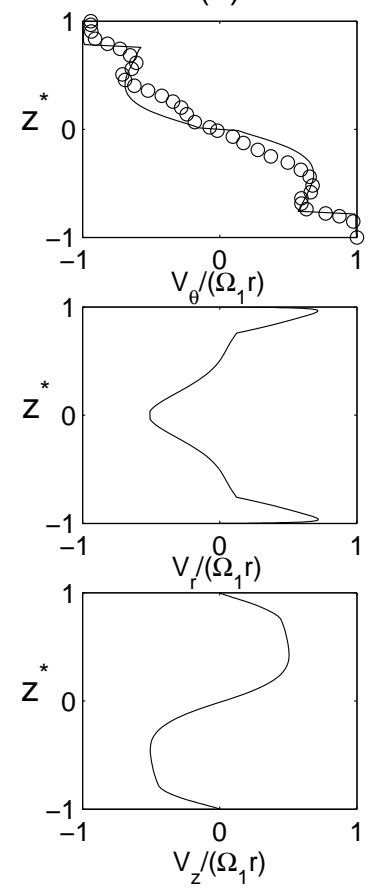

(b)
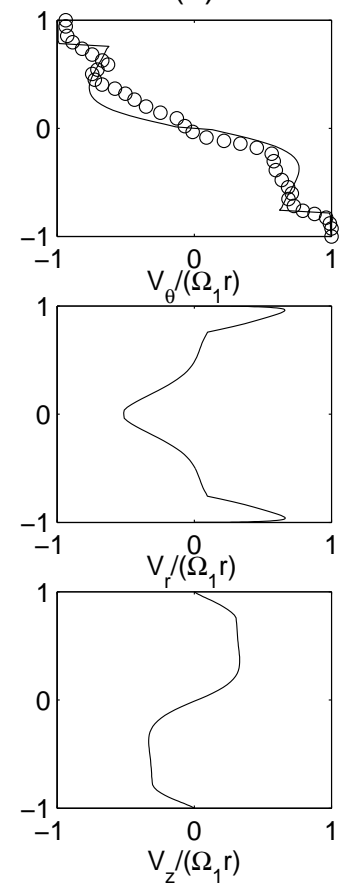

(c)
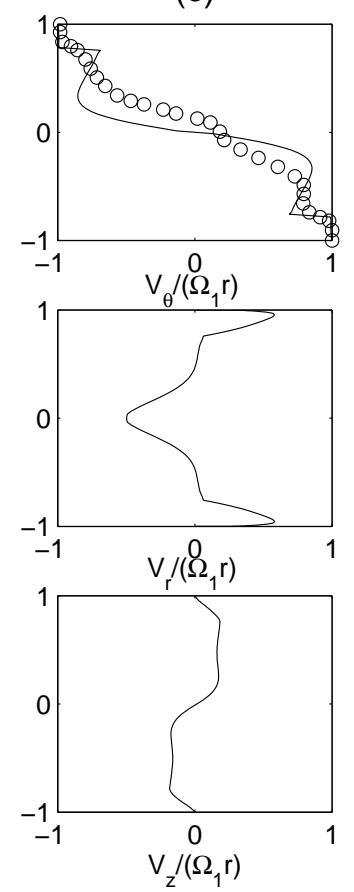

Figure 11: Poncet et al., submitted to Int. J. Heat Fluid Flow. 
(a)

(b)

(c)

(d)

(e)

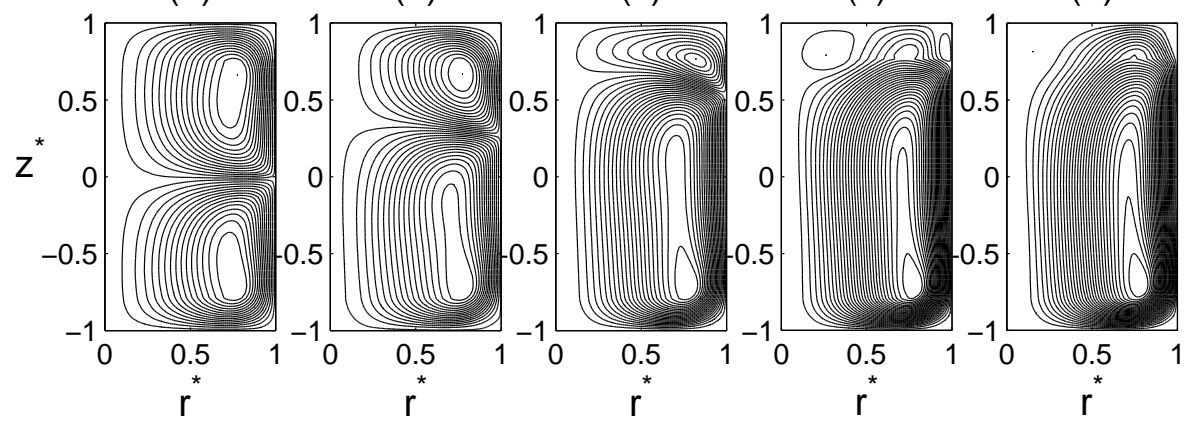

Figure 12: Poncet et al., submitted to Int. J. Heat Fluid Flow. 

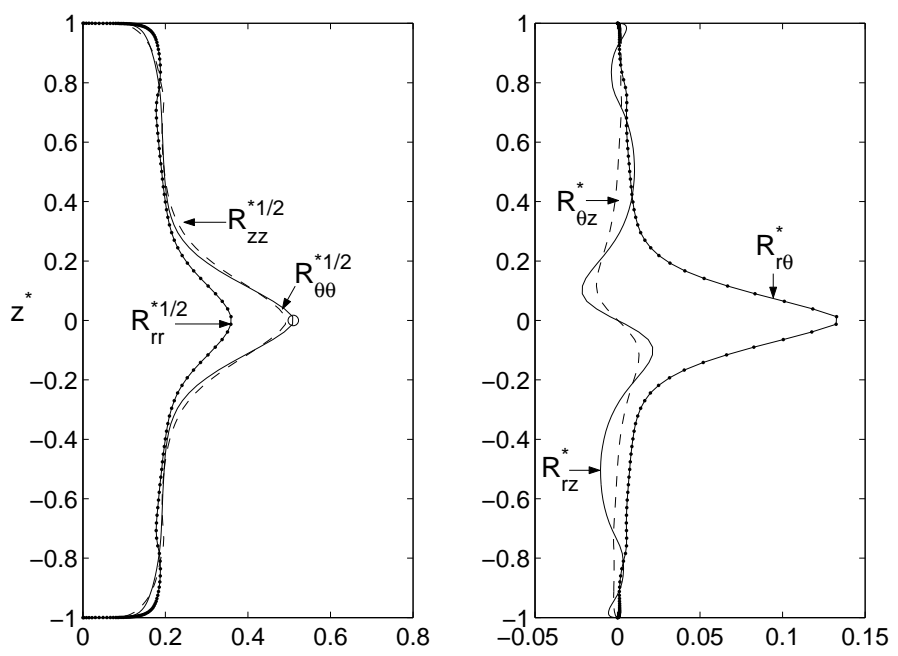

Figure 13: Poncet et al., submitted to Int. J. Heat Fluid Flow. 


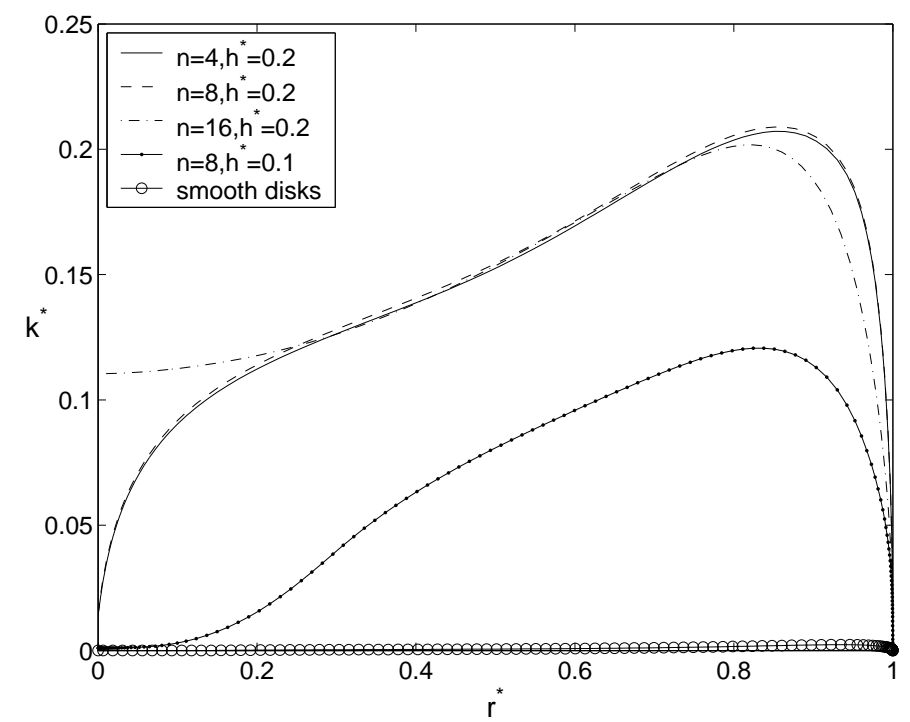

Figure 14: Poncet et al., submitted to Int. J. Heat Fluid Flow. 